\title{
Efecto de la Refinación Física Sobre el Aceite de la Almendra del Corozo (Acrocomia aculeata)
}

Carlos Hernández, Alberto Mieres, Zulay Niño y Sergio Pérez

Universidad de Carabobo, Facultad de Ingeniería, Escuela de Ingeniería Química, Avda. Bolívar N¹25-39, Valencia-Venezuela

(email: carherna@uc.edu.ve, albertopitre@icnet.com.ve, znino@uc.edu.ve,sperez@uc.edu.ve)

\section{RESUMEN}

El objetivo principal fue evaluar el efecto de la refinación física sobre las características del aceite de la almendra del fruto de la palma corozo (Acrocomia aculeata). La metodología se inició con un plan de muestreo que permitió obtener una muestra representativa del fruto del lugar de estudio. Se extrajo el aceite utilizando la operación de prensado en frío, luego se realizó una refinación física que consistió en un desgomado, blanqueado, desodorizado y desacidificación. Al aceite crudo y refinado se le determinó el perfil de ácidos grasos. En la etapa de desacidificación y desodorización el índice de peróxido fue de 1,974 miliequivalente de $\mathrm{O}_{2} \mathrm{~kg}^{-1}$ de aceite. El grado de acidez determinado como ácido oleico para el aceite crudo y refinado fue de 0,620 y $0,100 \%$, respectivamente. La refinación física aplicada redujo el olor y el grado de acidez de forma apreciable, pudiéndose recomendar para la purificación de este aceite.

\section{Effect of Physical Refining on the Oil of the Corozo's Kernel (Acrocomia aculeata)}

\begin{abstract}
The principal objective was to evaluate the effect of the physical refining on the characteristics in the oil of the corozo's palm kernel fruit (Acrocomia aculeata). The methodology, it began with a plan of sampling that allowed to obtain a representative sample of the fruit of the place of study. The oil was extracted using the operation of pressing in cold, then there was realized a physical refining that consisted of a degumming, bleaching, deodorized and desacidification. To the raw and refined oil there decided the profile of oily acids. In the stage of desacidification and deodorized the index of peroxide was of 1,974 miliequivalent of $\mathrm{O}_{2} \mathrm{~kg}^{-1}$ of oil. The degree of acidity determined as acid oleic for the raw and refined oil was of 0,620 and $0,100 \%$, respectively. The physical refining applied reduced the smell and the degree of acidity of valuable form, being able to be recommended for the purification of this oil.
\end{abstract}

Keywords: Acrocomia aculeata, physical refining, corozo palm, oily acids, degree of acidity 


\section{INTRODUCCIÓN}

La palma corozo se encuentra en todo el trópico Americano, se extiende desde el sur de México, a través de la América Central, Las Antillas, Bolivia, Paraguay y el norte de Argentina (Francis, 1993). Normalmente habita en sabanas abiertas o en tierras desforestadas, son muy frecuentes en áreas y campos perturbados y con épocas de lluvias bien definidas. El corozo también crece y se reproduce en los bosques húmedos de tierras bajas que reciben entre 1000 y $1900 \mathrm{~mm}$ de precipitación anual. El hábitat más favorable para la reproducción del corozo, que le provee a su vez de una ventaja competitiva en el crecimiento, son las arenas costeras húmedas. Estas son arenas margosas con un $\mathrm{pH}$ entre 6.5 y 8.5. Otros sitios que sostienen al corozo son los valles abiertos, los bancos de los ríos y los pastizales de los cerros húmedos. El corozo rara vez crece en las cuestas más altas o la cima de los cerros. Según Braun y Delascio (1987), la palma corozo (Acrocomia aculeata) se encuentra extendida por todo el territorio venezolano, encontrándose abundantemente en los estados Zulia, Bolívar, Carabobo, Cojedes, Lara y Portuguesa.

En Venezuela se han venido realizando de forma aislada, algunas investigaciones sobre el uso potencial de los frutos de algunas palmas autóctonas, de diferentes regiones del país, como posibles fuentes de alimentos. En este sentido, Belén et al. (2005b) evaluaron fisicoquímicamente el aceite crudo extraído con n-hexano de la almendra del fruto del corozo (Acrocomia aculeata), el cual fue recolectado en "El Chaparro" estado Anzoátegui, determinando a través del perfil de ácidos grasos, su uso potencial en la industria aceitera una vez refinado o purificado. Arveláez et al. (2006) aplicaron un diseño experimental para la extracción de aceite de la almendra del corozo recolectado en el estado Carabobo, evaluando la extracción por lixiviación con n-hexano y arrastre con vapor e infiriendo igualmente sobre la utilidad de este tipo de aceite en la industria alimenticia, una vez refinado. Belén et al. (2005a) caracterizaron el aceite obtenido de la almendra de la coroba (Jessenia polycarpa Karst), encontrando un gran contenido de ácido láurico. González (2004), obtuvo y caracterizó al aceite crudo del endospermo de la palma yagua (Attalea spp).

De acuerdo al boletín estadístico de la Asociación Venezolana de cultivadores de Palma Aceitera (2006), en el año 2005, Venezuela importó más de 400 mil toneladas de aceites y grasas vegetales, donde más de 62 mil toneladas correspondieron a la importación de aceite crudo y refinado de palma; y de palmiste.

Ante esta realidad se plantea la necesidad de incentivar la producción de rubros poco explotados con los cuales se podría competir exitosamente. Uno de ellos se basa en la extracción y refinación de aceites a partir de fuentes naturales no convencionales, tal es el caso del aprovechamiento del corozo (Acrocomia aculeata), el cual es una palma con una serie de cualidades que la hacen atractiva tanto para su uso industrial como alimenticio.

El objetivo principal de esta investigación, fue evaluar el efecto de la refinación física sobre las características del aceite de la almendra del fruto de la palma corozo (Acrocomia aculeata), determinando para ello el grado de acidez, el índice de peróxido, el rendimiento másico en cada etapa de la refinación física y el perfil de ácidos grasos en el aceite crudo y refinado.

\section{METODOLOGÍA}

\section{Muestras y lugar de recolección del fruto}

El área seleccionada es el estado Portuguesa, específicamente el sitio de recolección "La Aduana" a $80 \mathrm{~km}$ de Guanare. La recolección de los frutos se efectuó en el mes de enero, época de verano en dicho estado. Con la visita al área se recabó información para determinar el tamaño de la plantación (población) y el tamaño de la muestra, siguiendo un procedimiento aleatorio simple. Se recolectaron los frutos correspondientes a cada palma, luego se mezclaron en un saco, de manera de obtener una muestra representativa de la población. De acuerdo a Salas (1992), el estado Portuguesa es importante para el desarrollo de los cultivos de oleaginosas, ya que es una región que presenta valores pluviométricos promedios entre 1.400 y $1.500 \mathrm{~mm} / \mathrm{año}$, ocurriendo las máximas precipitaciones durante los meses de julio y agosto; en cambio presenta una época seca de 
aproximadamente cuatro meses, entre enero y abril. La temperatura promedio anual es de $27^{\circ} \mathrm{C}$, poco oscilante; con una insolación promedio de 2.100 horas luz/año y $84 \%$ de humedad relativa. Los suelos son planos, con pendiente inferior a $0,5 \%$, mal drenaje; ricos en nutrimentos y $\mathrm{pH}$ alrededor de 6. Estas características son precisamente las necesarias para el crecimiento y desarrollo de la palma corozo, de allí la importancia en la selección del estado Portuguesa como región para el estudio de la extracción del aceite de la almendra de este fruto.

\section{Muestreo}

Con el tamaño de la población y la definición del error, el cual fue considerado en $6 \%$, se determinó el tamaño de la muestra, la cual fue de 189 palmas de corozo. Por cada elemento de la muestra se recolectaron 25 frutos, los cuales fueron tomados directamente de las palmas, colocados y mezclados aleatoriamente en sacos, para su transporte al lugar donde se realizó la investigación.

\section{Extracción y molienda de la almendra del fruto de la palma corozo}

Una vez obtenida la materia prima para el análisis, la misma se sometió a un secado natural al sol, por 5 días, a una temperatura y humedad de $25^{\circ} \mathrm{C}$ y $60 \%$ respectivamente. Realizado el secado, se pesó el fruto y se procedió a la extracción de cada elemento constituyente del mismo. Posteriormente, se fracturó el epicarpo con la ayuda de un martillo, separándolo del resto del fruto. Las demás partes, conformadas por el mesocarpo, endocarpo y almendra, se sometieron a una segunda extracción mecánica, fracturando de esta manera al mesocarpo y facilitando la extracción manual de las almendras, las cuales se pesaron y almacenaron en recipientes limpios por una semana, en un ambiente refrigerado a $7^{\circ} \mathrm{C}$. A las almendras se les realizó una reducción de tamaño mediante un molino mecánico del cual se obtuvieron diferentes tamaños de semilla $(0,70$ a 0,80$) \mathrm{mm}$.

\section{Extracción del aceite}

El aceite se extrajo mediante un prensado en frío. Las presiones a las que se realizaron los ensayos fueron $2.000,4.000,6.000,8.000$ y 10.000 psi, durante un tiempo de 5,15 y 30 minutos, dos pruebas por tratamiento. Todos los ensayos fueron realizados con una muestra de almendra molida de 20 gramos aproximadamente y un tamaño de partícula comprendido entre 0,70 y 0,80 mm. El procedimiento consistió en colocar la muestra de almendra en un cilindro extractor, luego el mismo se sometió lentamente a la presión hasta alcanzar la requerida. Con la ayuda de la palanca de activación se mantuvo la presión constante durante el tiempo correspondiente para el ensayo, el cual se controló mediante el uso de un cronómetro. El aceite se recogió en un beacker de $10 \mathrm{~mL}$, pesado previamente. Culminado el tiempo de extracción se accionó la válvula que alivia la presión en la prensa hidráulica. La torta de prensado remanente que se encontraba en el cilindro extractor fue reprensada a la misma presión durante el mismo tiempo, con la finalidad de extraer aun mayor cantidad de aceite. El rendimiento total de la operación se determinó a partir de la masa de aceite obtenida del prensado y reprensado entre la cantidad de muestra de almendra utilizada.

\section{Refinación física}

Las etapas del proceso fueron las siguientes: desgomado, blanqueado y por último desacidificación y desodorizado, las cuales se realizaron en conjunto. Por otra parte, aun cuando todos los aceites crudos obtenidos por prensado en frío a las diferentes presiones y tiempos de procesamiento, son iguales, ya que provienen de las mismas muestras de almendras, el aceite que se utilizó para la refinación física fue el que se extrajo de la mejor condición del prensado aplicado.

\section{Desgomado}

El objetivo fue eliminar los fosfátidos o fosfolipidos presentes con una mínima pérdida de aceite neutro. La operación de desgomado fue realizada de acuerdo a lo propuesto por Nasirullah y Ramanatham (2000) en su investigación sobre la refinación física del aceite soya y salvado de arroz, en la que sugieren un proceso de desgomado en dos etapas para eliminar los fosfátidos hidratables y no hidratables. La primera etapa consistió en lavar con agua al aceite, para eliminar todos aquellos 
fosfolipidos hidratables como por ejemplo la lecitina. El aceite se calentó hasta alcanzar una temperatura cercana a los $60^{\circ} \mathrm{C}$, en ese momento se le adicionó $3 \% \mathrm{p} / \mathrm{p}$ de agua destilada, la cual se calentó previamente hasta una temperatura aproximada de $60^{\circ} \mathrm{C}$, antes de adicionarla al aceite. La mezcla se dejó con agitación constante por 30 minutos a una temperatura entre $60-70{ }^{\circ} \mathrm{C}$. Finalizado el tiempo, se dejó enfriar, se centrífugó a 3.500 rpm por 30 minutos para separar el aceite de los fosfolipidos hidratables.

Siguiendo la metodología de Nasirullah y Ramanatham (2000), se realizó la segunda etapa del desgomado, que consistió en eliminar los fosfolipidos no hidratables, los cuales son sales de calcio y magnesio del ácido fosfórico que son más solubles en aceite que en agua y por ende no se hidratan. Al usar un ion monovalente como el potasio, que sustituya a los iones divalentes calcio y magnesio, hace que las sales complejas no hidratables se conviertan a especies hidratables, debido a que sus sales son más solubles en agua. El aceite obtenido en la primera etapa fue tratado con una solución de cloruro de potasio al 1\% y en una relación muestra de aceite / cloruro de potasio de 98:2. La mezcla se calentó con agitación constante a una temperatura entre $60-70{ }^{\circ} \mathrm{C}$ por 15 minutos y se centrifugó a 3.500 rpm por 30 minutos para separar los fosfolipidos no hidratables.

\section{Blanqueado}

El objetivo de este proceso fue el de descomponer los peróxidos, eliminar los compuestos oxidantes y cualquier vestigios de gomas y jabones, y decolorar. El proceso de blanqueo se realizó con el uso de tierras de blanqueo, específicamente Tonsil grado óptimo, la cual se obtuvo de una empresa aceitera de la ciudad de Valencia. Se siguió el procedimiento propuesto por Nasirullah y Ramanatham (2000), en el cual el aceite desgomado se calentó hasta una temperatura aproximada de $80^{\circ} \mathrm{C}$, en ese momento se le adicionó $3 \%$ de la tierra de blanqueo antes mencionada, la cual estuvo 2 horas en la estufa a $80^{\circ} \mathrm{C}$ para eliminar cualquier vestigio de humedad que pudiera afectar el proceso. La mezcla de aceite y tierra se mantuvo con agitación y vacío constantes por 15 minutos a una temperatura entre $80-90^{\circ} \mathrm{C}$ y $60 \mathrm{~mm} \mathrm{Hg}$. La mezcla se dejó enfriar a temperatura ambiente, se centrifugó a 3.500 rpm por 30 minutos para separar el aceite de las tierras de blanqueo.

\section{Proceso de desacidificación y desodorización}

El objetivo de esta etapa fue reducir los ácidos grasos libres, producir un aceite con poco o ningún sabor y un color tenue. El proceso consistió en someter al aceite a una destilación por carga al vacío, de manera de eliminar los ácidos grasos libres, cetonas y aldehídos, los cuales poseen punto de ebullición más bajos que el resto de los triglicéridos presentes en el aceite. La desacidificación y desodorización se realizaron de acuerdo a la metodología propuesta por Pasarelli (1986), y consistió en tomar el aceite desgomado y blanqueado e introducirlo en una balón de dos bocas, que a su vez se colocó en una cuba que contenía glicerina como medio de calentamiento. La glicerina se calentó hasta una temperatura entre $100-120^{\circ} \mathrm{C}$, mediante una plancha eléctrica. Una de las bocas del balón que contiene el aceite se le colocó un termómetro, el cual indicaba la temperatura del mismo. La otra boca del balón fue conectada a un condensador y a su vez éste a la manguera de la línea de vacío. La operación antes indicada se realizó durante 3,5 horas con vacío continuo entre 4 y $6 \mathrm{~mm}$ Hg. Finalizado el tiempo, el balón fue retirado del baño de glicerina y colocado en otra cuba con agua a temperatura ambiente, de manera de lograr un rápido enfriamiento, todo esto sin desconectar el sistema del vacío, de forma tal de no permitir la entrada de aire.

\section{Rendimiento del refinado físico}

La cuantificación de la cantidad de aceite obtenido se realizó por gravimetría antes y después de cada etapa del proceso de refinación, es decir, se pesa la cantidad inicial de aceite antes de ser sometido a la etapa de refinado correspondiente y luego de cumplida la misma, de esta forma se logró determinar el rendimiento de cada etapa y del proceso completo de refinación.

\section{Perfil de ácidos grasos}

El perfil de ácidos grasos se realizó siguiendo la metodología establecida en la norma COVENIN 2281 (2002), la cual consistió en tomar una muestra de 300mg de aceite, se disolvió en $\mathrm{NaOH} \mathrm{0,5} \mathrm{N,}$ 
en metanol anhidro, se colocó en reflujo hasta que se disolvió el aceite y luego se añadió trifloruro de boro en metanol, se dejó a reflujo por 20 minutos. Se realizó la extracción con aproximadamente 100 $\mathrm{mL}$ de un solvente orgánico (n-heptano o cloroformo). La fase orgánica se utilizó para realizar la cromatografía de los ésteres de metilo. Se empleó un cromatógrafo HP 5890 con detector de ionización a la llama e integrador, una columna capilar DB-23 (Cat J \& W Scientific. US1363243H) de 30 centímetros, diámetro interno $0,32 \mathrm{~mm}$, con gas de arrastre nitrógeno y flujo de $82 \mathrm{~mL} / \mathrm{min}$. El gradiente de temperatura de $150-180^{\circ} \mathrm{C}$ con rata de $1^{\circ} \mathrm{C} / \mathrm{min}$. Se inyectaron 0,2 microlitros de muestra. Bajo las mismas condiciones se inyectaron los patrones de ésteres de los ácidos grasos. Por medio de las áreas correspondientes a cada éster se obtuvo la composición de ácidos grasos del aceite. El ensayo se realizó por triplicado, calculando el valor promedio y la desviación estándar.

\section{Variables de control utilizadas durante las etapas de la refinación física}

Las variables utilizadas para el control de cada una de las etapas de la refinación física del aceite crudo de la almendra, fue el grado de acidez y el índice de peróxidos, ya que estos pueden medir de manera cuantitativa el deterioro de la calidad del aceite. Los ensayos se realizaron por triplicado.

\section{Determinación del grado de acidez}

Este método esta fundamentado en la norma COVENIN 0325 (2001) y se basó en la reacción de neutralización de los ácidos grasos con una base. Para ello se preparó una solución alcohólica del aceite y se tituló con hidróxido de sodio. El grado de acidez por lo general se expresa como ácido oleico, sin embargo, puede expresarse como ácido palmítico o ácido láurico.

\section{Determinación del índice de peróxido}

Este índice esta basado en la capacidad del oxígeno peroxídico de oxidar el ión yoduro del yoduro de potasio (KI) produciendo yodo, el cual se valoró con una solución de tiosulfato de sodio. Considerando a los peróxidos como producto de la oxidación de las grasas, se puede tomar como la capacidad que tiene el aceite de continuar oxidándose. Para realizar esta prueba se siguió la norma COVENIN 0508 (2001). Se pesó aproximadamente 5 gramos de aceite, el cual se diluyó en una solución ácido acético-cloroformo y se hizo reaccionar con yoduro de potasio por un minuto, luego se le adicionó gran cantidad de agua y al observar cambio de color, se tituló con tiosulfato de sodio, la prueba también fue realizada sin muestra para obtener el blanco respectivo.

\section{RESULTADOS Y DISCUSIÓN}

\section{Peso del fruto y almendra del corozo}

La masa promedio del fruto del corozo fue de $22,42 \mathrm{~g}$ y el de la almendra de $2,53 \mathrm{~g}$, lo que corresponde a $11,28 \%$ con respecto al total. Arveláez et al. (2006) en sus trabajos con el corozo del estado Carabobo, determinaron que el porcentaje de la almendra es de $12 \%$, lo que indica que el fruto de ambos regiones presentan similitud en cuanto al peso de este elemento.

\section{Prensado en frío}

La tabla 1, contiene los rendimientos de la extracción de aceite crudo de almendra, obtenidos por prensado en frío. Se observa que el rendimiento se incrementó con el tiempo de prensado en cada una de las presiones evaluadas. Igual tendencia se observó con la presión de prensado desde 2.000 psi hasta 8.000 psi, donde se estabilizó. En este sentido Mpagalile y Clarke (2005), reportaron en su investigación sobre la eficiencia del prensado de aceite de coco, un valor de presión (13 MPa) en la que no se producen igualmente cambios importantes en este parámetro. Por otra parte, es notorio destacar que entre 8.000 y 10.000 psi, no se presentaron diferencias apreciables en el rendimiento para un tiempo de prensado de 30 minutos, lo que se explica por el hecho de que transcurrido cierto tiempo y presión, el sedimento se hace incompresible, de modo que ya no es posible continuar la operación de prensado (Mafart y Béliard, 1994). De acuerdo a esto, la mejor condición para la 
extracción de aceite por prensado en frío fue de 8.000 psi y 30 minutos y la que finalmente se usó para obtener la cantidad de aceite crudo necesario para realizar la refinación física propuesta.

Tabla 1: Rendimiento de la extracción de aceite de la almendra del corozo por prensado en frío

\begin{tabular}{|c|c|c|}
\hline $\begin{array}{c}\text { Presión } \\
\text { psi }\end{array}$ & $\begin{array}{c}\text { Tiempo } \\
\text { min. }\end{array}$ & $\begin{array}{c}\text { Rendimiento total } \\
\%\end{array}$ \\
\hline \multirow{3}{*}{2.000} & 5 & 5,79 \\
\cline { 2 - 3 } & 15 & 7,45 \\
\cline { 2 - 3 } & 30 & 10,49 \\
\hline \multirow{3}{*}{4.000} & 5 & 9,24 \\
\cline { 2 - 3 } & 15 & 13,17 \\
\cline { 2 - 3 } & 30 & 14,06 \\
\hline \multirow{3}{*}{6.000} & 5 & 11,04 \\
\cline { 2 - 3 } & 15 & 13,57 \\
\hline \multirow{3}{*}{8.000} & 30 & 15,11 \\
\cline { 2 - 3 } & 5 & 13,01 \\
\cline { 2 - 3 } & 15 & 16,44 \\
\hline \multirow{3}{*}{10.000} & 30 & 22,23 \\
\cline { 2 - 3 } & 5 & 14,53 \\
\cline { 2 - 3 } & 15 & 16,01 \\
\hline
\end{tabular}

\section{Rendimientos obtenidos en las diferentes etapas de la refinación física}

La tabla 2, muestra los rendimientos de las etapas del refinado físico del aceite de la almendra del fruto de la palma corozo, así como el rendimiento total del proceso. Las mermas o pérdidas del primer tratamiento del desgomado, correspondieron a los fosfolipidos hidratables eliminados y el aceite neutro que éstos arrastran. El segundo tratamiento, el cual consistió en la eliminación de los fosfolipidos no hidratables, el rendimiento obtenido fue menor, esto se debió a que los floculos formados fueron de menor densidad que los floculos del primer tratamiento, lo que dificulta su separación del aceite neutro, ocurriendo de esta forma mayor arrastre. Sin embargo, el rendimiento total del proceso de desgomado con los tratamientos antes planteados, resultó superior en comparación con procesos de desgomado ácidos y básicos, en la cual se realizan numerosos lavados con agua para reducir la acidez del aceite neutro resultante, lo que representa una ventaja comparativa en este aspecto, tal como lo indica Nasirullah (2005) en su estudio sobre el desgomado de gomas no hidratables en aceites vegetales.

Las pérdidas ocurridas en la etapa de blanqueo fueron debido a la retención de aceite por parte del agente de blanqueo utilizado, sin embargo fueron mucho menor que las mermas que se pudieran obtener por usar otros agentes como el carbón activado, que aun siendo mejor en la reducción de color y olor en los aceites, producen mayores mermas y bajo rendimiento, según lo reportado por Nasirullah y Ramanatham (2000).

En la etapa de desacidificación y desodorización las pérdidas fueron pequeñas $(0,71 \%)$, ya que en ésta etapa se eliminan ácidos grasos libres, sustancias volátiles e insaponificables, causantes del olor y sabor de los aceites. Aparece reportado en la literatura (Andersen, 1956), que las pérdidas en la desodorización de los aceites de coco, girasol, algodón, palma y palmiste alcanzan un 0,1-0,3\% de la grasa original. Sin embargo las pérdidas en la desodorización del aceite de la almendra de corozo fueron superiores, debido quizás al arrastre de aceite neutro durante la destilación. Por otra parte la desacidificación física, presenta mayores rendimientos que la desacidificación o neutralización alcalina, también llamada refinación química, donde el rendimiento no alcanza valores superiores al $60 \%$, siendo esto una ventaja importante de la refinación física con respecto a la química. En este mismo sentido Rossi et al. (2001), en sus investigaciones sobre el efecto del blanqueo y refinación física en el color y componentes menores del aceite de palma, sugieren que la refinación física es 
preferida antes que la química, ya que esta última es causante de excesivas pérdidas de aceite durante el proceso.

Tabla 2: Rendimientos de las etapas del refinado físico

\begin{tabular}{|l|l|c|}
\hline \multicolumn{2}{|c|}{$\begin{array}{c}\text { Etapas del proceso de } \\
\text { refinación física }\end{array}$} & Rendimiento (\%) \\
\hline \multirow{4}{*}{ Desgomado } & $\begin{array}{l}\text { Primer } \\
\text { tratamiento }\end{array}$ & 93,83 \\
\cline { 2 - 3 } & $\begin{array}{l}\text { Segundo } \\
\text { tratamiento }\end{array}$ & 89,32 \\
\cline { 2 - 3 } & Total & 83,80 \\
\hline Blanqueado & 91,49 \\
\hline $\begin{array}{l}\text { Desacidificación/ } \\
\text { Desodorización }\end{array}$ & 99,29 \\
\hline Refinado & 76,12 \\
\hline
\end{tabular}

Perfil de ácidos grasos

En la tabla 3, se presenta el valor promedio $(X)$ y la desviación estándar $(\sigma)$ de los ácidos grasos del aceite crudo y refinado extraído de la almendra del fruto de la palma corozo. Allí se observa que tras la aplicación de la refinación física y de acuerdo al tratamiento estadístico de los datos experimentales, no se aprecian diferencias significativas en el porcentaje de los ácidos grasos presentes en el aceite $(P>0,05)$, para un nivel de significación del $5 \%$.

Tabla 3: Perfil de ácidos grasos del aceite crudo y refinado extraído de la almendra del corozo

\begin{tabular}{|c|c|c|}
\hline Ácidos grasos & $\begin{array}{c}\text { Aceite crudo } \\
(\mathrm{X} \pm \sigma) \%\end{array}$ & $\begin{array}{c}\text { Aceite refinado } \\
(\mathrm{X} \pm \sigma) \%\end{array}$ \\
\hline Caprílico $(\mathrm{C} 8: 0)$ & $5,81 \pm 0,22$ & $6,59 \pm 0,63$ \\
\hline Cáprico $(\mathrm{C} 10: 0)$ & $3,69 \pm 0,27$ & $4,31 \pm 0,46$ \\
\hline Láurico (C12:0) & $45,00 \pm 2,95$ & $51,00 \pm 2,72$ \\
\hline Mirístico (C14:0) & $12,82 \pm 0,27$ & $12,97 \pm 0,19$ \\
\hline Palmítico (C16:0) & $7,78 \pm 0,77$ & $6,81 \pm 0,28$ \\
\hline Esteárico (C18:0) & $3,09 \pm 0,86$ & $2,12 \pm 0,71$ \\
\hline Oleico (C18:1) & $18,67 \pm 2,33$ & $14,00 \pm 2,41$ \\
\hline Linoleico (C18:2) & $3,14 \pm 0,92$ & $2,20 \pm 0,40$ \\
\hline
\end{tabular}

De acuerdo al perfil de ácidos grasos del aceite refinado extraído de la almendra del corozo, utilizando la técnica de prensado en frío, se puede inferir que es muy similar al aceite de coco y al de la almendra del fruto de la palma africana (palmiste), en cuanto al contenido de ácidos grasos saturados e insaturados y a la proporción de ácido láurico presente. Canapi et al. (2005) y Salas (1992), reportan el perfil de ácidos grasos del aceite de coco y palmiste respectivamente, el cual se observa en la tabla 4.

Por otra parte, es importante señalar que Belén et al. (2005b) en sus investigaciones sobre la caracterización del aceite crudo de la almendra del corozo de una zona del estado Anzoátegui, determinaron que este aceite es rico en ácidos grasos saturados $(79,6 \%)$, donde predomina el ácido láurico con $50,9 \%$.

Otras investigaciones, como las realizadas por Arveláez et al. (2006) con la almendra del corozo de una región del estado Carabobo y por González (2004) con el fruto de la palma yagua, indican la presencia del ácido láurico como ácido graso mayoritario presente en el aceite con $56,6 \%$ y 58,0\% respectivamente. 
Tabla 4: Ácidos grasos en el aceite de coco y palmaste

\begin{tabular}{|c|c|c|}
\hline Ácidos grasos & $\begin{array}{c}\text { Aceite de } \\
\text { coco }(\%)\end{array}$ & $\begin{array}{c}\text { Aceite de } \\
\text { palmiste }(\%)\end{array}$ \\
\hline Caprílico (C8:0) & $5,4-7,9$ & $3,0-6,0$ \\
\hline Cáprico (C10:0) & Trazas -0,8 & $3,0-6,0$ \\
\hline Láurico (C12:0) & $45,4-48,0$ & $50,0-52,0$ \\
\hline Mirístico (C14:0) & $17,5-18,0$ & $15,0-16,0$ \\
\hline Palmítico (C16:0) & $9,0-10,5$ & $6,5-7,5$ \\
\hline Esteárico (C18:0) & $2,1-2,3$ & $1,0-1,5$ \\
\hline Oleico (C18:1) & $5,7-7,5$ & $16,0-16,5$ \\
\hline Linoleico (C18:2) & $0,0-2,6$ & $1,0-1,5$ \\
\hline
\end{tabular}

El ácido graso que se encontró en el aceite refinado de la almendra del corozo en el nivel más alto fue el láurico $(51,00 \pm 2,72) \%$, lo cual le confiere propiedades importantes para aplicaciones alimenticias, debido a que son de bajo peso molecular y bajo grado de insaturación, por lo que constituyen valiosas grasas comestibles para ciertas finalidades. En este sentido, Carr y Hogg (2005) analizaron las perspectivas de los fabricantes de productos basados en aceite de palma y palmiste, este último rico en ácido láurico; y la variedad de usos que se les puede dar en panaderías, elaboración e imitación de cremas, cremas vegetales, emulsiones alimenticias, entre otros, pudiéndose recomendar el aceite refinado de la almendra del corozo como sustituto o complemento del aceite de palmiste, en los productos antes señalados.

Con respecto al potencial nutricional de los aceites ricos en ácido láurico (C12:0), como el de la almendra del corozo. De Roos et al. (2001), realizaron pruebas sobre la lipoproteína sérica en seres humanos y su efecto en el colesterol, comparando el consumo de grasas sólidas ricas en ácido láurico y grasas sólidas ricas en ácidos grasos trans. La investigación arrojó que el consumo de grasas con alto contenido en ácido láurico, es más favorable sobre el colesterol sérico total, que el consumo de grasas hidrogenadas ricas en ácidos grasos trans, como la obtenida a partir del aceite de soya.

\section{Variables de control utilizadas en cada etapa de la refinación física}

Se utilizaron como variables de control de la refinación física, las mediciones de grado de acidez e índice de peróxido. En la tabla 5 se presentan estos resultados. Allí se observa que no hubo oxidación del aceite de la almendra de corozo hasta la etapa de desodorización y desacidificación, en la que se incrementó, debido a las altas temperaturas desarrolladas en el proceso de destilación de las etapas antes mencionadas, sin embargo, el índice de peróxido se encuentra dentro del rango establecido por la norma venezolana COVENIN 0069 (2006) para los aceite comerciales, lo que demuestra una alta resistencia a la oxidación lipídica. El grado de acidez disminuye hasta un $84 \%$ en el aceite refinado en comparación con el aceite crudo. Aun cuando la mejora es apreciable, el mismo se encuentra en el límite superior que indica la misma norma, pudiendo sugerirse ajustes en el tiempo y temperatura de las etapas de desacidificación y desodorización.

La evaluación fisicoquímica del aceite crudo de la almendra del corozo, recolectada en "El Chaparro" estado Anzoátegui (Belén et al., 2005b) indica que no se detectó peróxido en el aceite después de la extracción con n-hexano, evidenciando una alta estabilidad frente a la temperatura. Por otra parte, el grado de acidez fue de $0,4 \%$ expresado como ácido oleico, siendo este valor superior a lo recomendado por la norma COVENIN 0069 (2006), que regula la calidad de los aceites y grasas vegetales comestibles y establece que no debe ser mayor a $0,1 \%$.

La operación de desacidificación y desodorización se realizó con la finalidad de remover materiales odoríferos volátiles y los ácidos grasos libres causantes del olor y sabor en los aceites. El aceite crudo de la almendra de corozo presenta un olor similar al del aceite de coco, lo que indica probablemente la presencia de metilnonilcetona, responsable del olor característico de dicho aceite. 
Después del proceso de desodorizado, el olor se reduce considerablemente, hasta el punto de ser casi imperceptible.

Tabla 5: Índice de peróxido y grado de acidez en el aceite de la almendra del corozo

\begin{tabular}{|c|c|c|}
\hline Condición & $\begin{array}{c}\text { Índice de peróxido } \\
\text { meq } \mathrm{O}_{2} \mathrm{~kg}^{-1} \text { de aceite }\end{array}$ & $\begin{array}{c}\text { Grado de acidez } \\
\% \text { de ácido oleico }\end{array}$ \\
\hline Aceite crudo & No se detectó & 0,620 \\
\hline Aceite desgomado & No se detectó & 0,650 \\
\hline Aceite blanqueado & No se detectó & 0,580 \\
\hline Aceite refinado & 1,974 & 0,100 \\
\hline
\end{tabular}

\section{CONCLUSIONES}

La extracción de aceite se realizó por prensado en frío, encontrándose que el rendimiento de esta operación se incrementó con el tiempo y con la presión de prensado hasta 8.000 psi, donde se estabilizó.

No se detectó peróxidos en el aceite crudo, ni en las etapas de desgomado y blanqueado, sin embargo, se incrementó en la desacidicación y desodorización, manteniéndose dentro de los límites recomendados, demostrando que el aceite tiene una elevada estabilidad a la temperatura.

Con la refinación física aplicada se redujo el olor y el grado de acidez del aceite de forma apreciable, encontrándose este último parámetro en el límite superior de la normativa nacional vigente, por lo que pudiera sugerirse la refinación física propuesta para purificar el aceite de la almendra del corozo y para fines industriales, haciendo ajustes en el tiempo y temperatura de procesamiento en la etapa de desacidificación y desodorización, seguido de una neutralización química suave.

El rendimiento másico total de la refinación física del aceite de la almendra del corozo fue adecuado y comparable con la refinación física de otros aceites. Las mermas o pérdidas ocurrieron por arrastre de aceite neutro en cada una de las etapas del proceso.

Después de la refinación no se aprecian cambios significativos en el perfil de ácidos grasos del aceite refinado con respecto al crudo $(P>0,05)$, determinado estadísticamente con un nivel de significación del $5 \%$. Por otra parte, el aceite de la almendra del corozo presenta una composición en ácidos grasos especifica de aceites láuricos, siendo recomendable su uso en productos alimentarios como sustituto de aceites de uso convencional como el de coco y palmiste.

\section{REFERENCIAS}

Andersen, J.; Refinación de Aceites y Grasas para Usos Alimenticios, $1^{\text {a }}$ edición, 161-165, Continental, Distrito Federal, México (1956).

Arveláez, A., A. Mieres y C. Hernández; Diseño Experimental Aplicado a la Extracción de Aceite de la Almendra del Corozo (Acrocomia aculeata), Actas del $4^{\text {to }}$ Congreso Internacional y $15^{\text {to }}$ Congreso Nacional de Ingeniería Bioquímica, p. 660, Morelia - México, Abril 4-7 (2006).

Asociación Venezolana de Cultivadores de Palma Aceitera; Boletín Estadístico Sector Oleaginosas, Aceites y Grasas del Año 2005, 3-7, Caracas, Venezuela (2006).

Belén, D. y otros cinco autores; Physical and Chemical Characteristics of Coroba (Jessenia polycarpa Karst) Fruit: a Venezuelan Oleaginous Specie, Grasas y Aceites, 56(4), 317-323 (2005a).

Belén, D. y otros cinco autores, Physicochemical Evaluation of Seed and Seed Oil of Corozo (Acrocomia aculeata Jacq.), Grasas y Aceites, 56(4), 311-316 (2005b). 
Braun, A. y F. Delascio; Palmas Autóctonas de Venezuela y de los Países Latinoamericanos, $1^{\text {a }}$ edición, 28-76, Litopar, Caracas, Venezuela (1987).

Canapi, E.C. y otros cinco autores; Coconut Oil, Bailey's Industrial Oil and Fat Products, $6^{\text {th }}$ edición, Wiley - Intersciencie, 2, pp. 110-147, New York - USA (2005).

Carr, NO. y W.F. Hogg, A Manufacturer's Perspective on Selected Palm-Based Products, Asia Pacific Journal of Clinical Nutrition, 14(4), $381-386$ (2005).

COVENIN 0069; Norma Venezolana sobre Grasas Vegetales Comestible o Mantecas Vegetales Comestibles, 1-8, Caracas, Venezuela (2006).

COVENIN 0325; Norma Venezolana sobre Aceites y Grasas Vegetales. Determinación de la Acidez, 1-6, Caracas, Venezuela (2001).

COVENIN 0508; Norma Venezolana sobre Aceites y Grasas Vegetales. Determinación del Índice de Peróxidos, 1-6, Caracas, Venezuela (2001).

COVENIN 2281; Norma Venezolana sobre Aceites y Grasas Vegetales. Determinación de Ácidos Grasos por Cromatografía de Gases, 1-10, Caracas, Venezuela (2002).

De Roos, N.M., E.G. Schouten y M.B. Katan; Human Nutrition and Metabolism: Consumption of a Solid Fat Rich in Lauric Acid Results in a More Favorable Serum Lipid Profile in Healthy Men and Women than Consumption of a Solid Fat Rich in Trans Fatty Acids, Journal of Nutrition, 131(2), 242 245 (2001).

Francis, J.; Acrocomia media O.F. CooK, Corozo Palmae, SO-ITF-SM-68, Department of Agricultura, Forest Service, Southern Forest Experiment Station, New Orleans, LA., U.S, 11-14 (1993).

González J.; Obtención y Caracterización del Aceite Crudo del Endospermo de la Palma Yagua, Trabajo de grado. Ingeniería de Alimentos, Universidad Nacional Experimental Simón Rodríguez, Canoabo, Venezuela (2004).

Mafart, P. y E. Béliard; Técnicas de Separación, Ingeniería Industrial Alimentaria, $1^{a}$ edición, Acribia S.A, 2, 80-175, Zaragoza, España (1994).

Mpagalile, J.J. y B. Clarke; Effect of Processing Parameters on Coconut Oil Expression Efficiencies, International Journal of Food Sciences and Nutrition, 56(2), 125-132 (2005).

Nasirullah K.; Electrolyte Degumming of Nonhydratable Gums From Selected Vegetable Oils, Journal of Food Lipids, 12(2), 103 - 111 (2005).

Nasirullah K. y G. Ramanatham, Physical Refining of Rice Bran and Soybean Oils. Journal Food Sci. Technol. 37(2), 135-138 (2000).

Passarelli, A.; Estudio de Alternativas en el Proceso de Refinación de Aceites Vegetales con la Finalidad de Obtener un Producto para Consumo Humano, Trabajo de Grado. Escuela de Ingeniería Química, Universidad de Carabobo, Valencia, Venezuela (1986).

Rossi, M. y otros tres autores; The Effect of Bleaching and Physical Refining on Color and Minor Components of Palm Oil, Journal of the American Oil Chemist Society, 78(10), 1051-1055 (2001).

Salas, R.; La Palma Aceitera Africana, $1^{\text {a }}$ edición, 18-58, Consejo de Desarrollo Científico y Humanístico, Caracas, Venezuela (1992). 